JHT Read for CRedit Article \#317.

Scientific/Clinical Article

\title{
Stronger relation between impairment and manual capacity in the non-dominant hand than the dominant hand in congenital hand differences; implications for surgical and therapeutic interventions
}

\author{
Monique S. Ardon MSc, PT ${ }^{a, b}, *$, Ruud W. Selles PhD ${ }^{a, b}$, Steven E.R. Hovius MD, PhD ${ }^{b}$, \\ Henk J. Stam MD, PhD ${ }^{a}$, Magdalena Murawska MSc ${ }^{c}$, Marij E. Roebroeck $\mathrm{PhD}^{\mathrm{a}}$, \\ Wim G.M. Janssen MD, PhD ${ }^{\mathrm{a}}$ \\ ${ }^{a}$ Department of Rehabilitation Medicine and Physical Therapy, Erasmus MC - University Medical Center Rotterdam, P.O. Box 2040, 3000 CA, Rotterdam, The Netherlands

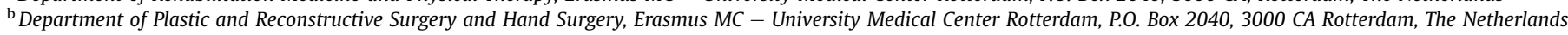 \\ ${ }^{\mathrm{c}}$ Department of Biostatistics, Erasmus MC - University Medical Center Rotterdam, P.O. Box 2040, 3000 CA Rotterdam, The Netherlands
}

\section{A R T I C L E I N F O}

\section{Article history:}

Received 14 March 2013

Received in revised form

12 November 2013

Accepted 20 November 2013

Available online 4 December 2013

\section{Keywords:}

Adolescent

Child

Female

Hand deformity

Congenital

Body functions of the hand and forearm

Male

Manual activity capacity

\begin{abstract}
A B S T R A C T
Objectives: To evaluate manual activity capacity (i.e. activity capacity to perform hand activities) and its relation with body functions of the hand and forearm in children with congenital hand differences (CHD) Methods: We assessed 10-14 year-old children with CHD $(N=106)$ using a functional handgrips test. Measurements of body functions included joint mobility and muscle strength. Patient characteristics were hand dominance and severity.

Results: We found a stronger relation between body functions and manual activity capacity in nondominant hands than dominant hands. Dominant hands scored significantly higher on manual activity capacity than nondominant hands that were similarly impaired at body functions level. Severity of the CHD and body functions had only small effects on manual activity capacity.

Conclusion: The relation between body functions and manual activity capacity is stronger in nondominant hands than dominant hands, indicating that improvement in body functions lead to larger changes in manual activity capacity in the non-dominant hand. This may suggest that in bilaterallyaffected children surgery should be done at the non-dominant hand first since this hand would benefit most from surgery-induced body functions improvement.
\end{abstract}

(C) 2014 Hanley \& Belfus, an imprint of Elsevier Inc. All rights reserved.
Abbreviation: $\mathrm{CHD}$, congenital hand difference.

This study was supported by Johanna Children's Fund (Arnhem, The Netherlands) and Kinderfonds Adriaanstichting (Rotterdam, The Netherlands) grant number 2006/0062-063. Their role was purely to give financial support for the study. The authors declare they have no competing financial interests.

We certify that no party having a direct interest in the results of the research supporting this article has or will confer a benefit on us or on any organization with which we are associated and, if applicable, we certify that all financial and material support for this research (e.g., NIH or NHS grants) and work are clearly identified in the title page of the manuscript.

* Corresponding author. Department of Rehabilitation Medicine, Erasmus MC University Medical Center Rotterdam, P.O. Box 2040, 3000 CA Rotterdam, The Netherlands. Tel.: +31 10 7044600; fax: +31 107033843 .

E-mail address: m.ardon@erasmusmc.nl (M.S. Ardon).

\section{Introduction}

The impact of a congenital hand difference (CHD) on a child's functioning can be described at different levels of functioning. ${ }^{1}$ The World Health Organization's International Classification of Functioning, Disability, and Health for Children and Youth (ICF-CY) distinguishes three domains: body functions, activity and participation. ${ }^{2}$ Children with a CHD can experience impairments in body functions of the hand and forearm, further referred to as body functions, such as restricted joint mobility, sensation and grip strength, and may be restricted in activities.

A child's activity, which the ICF-CY defines as the execution of a task or action by an individual, can be described by the two qualifiers of capacity and performance. 'Capacity' is defined as what a child can do in a standardized environment, and 'performance' is 
what a child actually does do in daily life. Impairments in body functions can lead to restrictions in capacity to perform daily activities that require the use of the upper limbs. ${ }^{3}$ While capacity reflects the child's ability to execute a task, performance is additionally influenced by the child's environment, which can facilitate or hamper performance. ${ }^{2}$

In general, rehabilitation interventions as well as surgical interventions in children with CHD aim to improve body functions with the ultimate goal to improve manual activity capacity, also referred to as dexterity or manual ability. For example, hand surgeons perform muscle tendon transfers to enhance strength for specific movements or perform osteotomies to improve alignment of bones and joint movements. Even so, strength training and splinting therapy aim to improve muscle strength and joint mobility. However, the relationship between body functions and manual activity capacity to perform tasks is not straightforward and therefore it is difficult to state whether these interventions lead to the intended improvement of manual activity capacity. 4,5

Although body functions in children with CHD are well studied, the key components of the domain of body functions determining manual activity capacity of these children are largely unknown. ${ }^{6-8}$ For example, many surgical interventions aim at strengthening the thumb, since it is assumed that the presence of a thumb accounts for at least $40 \%$ of the usefulness of the total hand. ${ }^{9}$ However, objective data are lacking to support which muscle functions are most important for manual activity capacity and which levels of joint mobility and muscle strength are needed for manual activity capacity.

Understanding the relation between body functions and manual activity capacity is essential for developing and selecting appropriate intervention strategies in children with CHD. Therefore, the aim of the present study was to disentangle the relationship between body functions and manual activity capacity in children with CHD.

\section{Materials and methods}

This study used data from a cross-sectional study on functioning and health-related quality-of-life of children with a CHD. The Medical Ethical Committee of our hospital approved the study and parents gave their informed consent to participate, as did all children above 12 years of age.

\section{Participants}

Participants in this study sample were recruited from a database of children with a CHD treated at our hospital. Inclusion criteria were: age 10-14 years, no cognitive or developmental delay, and sufficient knowledge of the Dutch language. We selected a heterogenous group of CHD patients to ensure that we had a relatively large variation in both body functions and manual activity capacity that would allow for investigating their interrelations. Three hundred participants were randomly selected using a computer generated random sequence. We evaluated 120 participants and we found no differences between participants and non-participants regarding gender, diagnosis, and severity of the CHD. Children and their parents received a letter concerning the purpose and procedure of the study. When they agreed to participate, a measurement session was planned and inform consent forms were signed. Due to time burden, some children did not participate in all measurements and we had missing values on some outcome measures (in 5 children we missed measurements on manual activity capacity, in 4 children on strength, in 1 child both on manual activity capacity and strength, in 2 on Kapandji and in 2 on palmar abduction) Therefore, we were able to evaluate 106 of the children
Table 1

Characteristics of participating children

\begin{tabular}{lc} 
Age (mean \pm SD, range) & $11.8 \pm 1.6$ \\
Gender & $(10-14)$ years \\
Boys & $55 \%$ \\
Girls & $45 \%$ \\
Affected side & \\
Unilateral & $69 \%$ \\
$\quad$ Nondominant affected & $62 \%$ \\
$\quad$ Dominant affected & $7 \%$ \\
Bilateral (Nondominant and dominant affected) & $31 \%$ \\
Extent of the CHD (affected digits per hand) & \\
1 & $29 \%$ \\
2 & $11 \%$ \\
3 & $12 \%$ \\
4 & $10 \%$ \\
5 & $37 \%$ \\
Surgical treatment & \\
None & $36 \%$ \\
1 or more & $64 \%$ \\
Diagnosis according to IFSSH classification & \\
Failure of formation & $25 \%$ \\
Failure of differentiation or separation of parts & $21 \%$ \\
Duplication & $21 \%$ \\
Overgrowth & $1 \%$ \\
Undergrowth & $29 \%$ \\
Congenital constriction ring syndrome & $3 \%$ \\
Generalized skeletal abnormalities & $0 \%$ \\
\hline
\end{tabular}

for the mentioned research purpose. Characteristics of the participating children are presented in Table 1.

\section{Manual activity capacity}

Since there is currently no available standardized assessment of manual activity capacity in children with CHD, we tested manual activity capacity using the Eliasson test. Eliasson et al developed this test for children based on the Sollerman test, evaluating 6 types of grip in 9 tasks (Table 2). ${ }^{10}$ This test, which is less extensive than the Sollerman test and more suitable for children of our age group, consists of tasks that require grasping objects either transverse grasping or diagonal grasping and tasks that require pinch grip. All tasks are scored on a 5-level ordinal scale. The scores range from 0 if the child cannot grip the object to 4 if the child can grip the object

Table 2

Eliasson test for manual activity capacity; tasks and scoring system

\begin{tabular}{|c|c|}
\hline \multicolumn{2}{|l|}{ Type of grasp } \\
\hline Transverse grasp & $\begin{array}{l}\text { Grasp a } 2.5 \mathrm{~cm} \text { horizontal bar in mid-air and place it on } \\
\text { the table }\end{array}$ \\
\hline Transverse grasp & $\begin{array}{l}\text { Move a } 2.5 \mathrm{~cm} \emptyset \text { vertical bar from one pegboard } \\
\text { position to another }\end{array}$ \\
\hline Transverse grasp & Lift a glass and pretend to drink \\
\hline Diagonal grasp & Hold a knife and cut paste into pieces \\
\hline Five-finger pinch & Pull a sleeve on and off the unaffected arm \\
\hline Tripod pinch & Unscrew a $2 \mathrm{~cm} \varnothing$ cap from a toothpaste tube \\
\hline Tripod pinch & Unscrew a $7 \mathrm{~cm} \emptyset$ lid from a jar \\
\hline Lateral pinch & $\begin{array}{l}\text { Grasp a vertically oriented plate }(5 \times 5 \times 1 \mathrm{~cm}) \text { in } \\
\text { mid-air and place it on a table; requiring supination } \\
\text { of the forearm }\end{array}$ \\
\hline Pinch & Pick up a small cube and touch the chin with it \\
\hline Score & Judgment of grips \\
\hline 0 & Cannot grip the object \\
\hline 1 & Grips object but cannot complete task \\
\hline 2 & $\begin{array}{l}\text { Grips object using an awkward grip and motion but } \\
\text { completes task }\end{array}$ \\
\hline 3 & $\begin{array}{l}\text { Grips object using a slightly deviant grip and motion but } \\
\text { completes task }\end{array}$ \\
\hline 4 & $\begin{array}{l}\text { Grips object using normal grip and motion and } \\
\text { completes task }\end{array}$ \\
\hline
\end{tabular}


Table 3

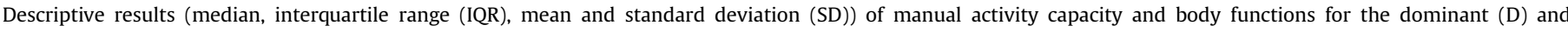
nondominant (ND) hand; absolute values and percentage of norm values

\begin{tabular}{|c|c|c|c|c|c|c|}
\hline & & Me (IQR) & Mean (SD) & $\begin{array}{l}\% \text { of norm } \\
\text { Me (IQR) }\end{array}$ & $\%$ of norm Mean (SD) & $p$-value \\
\hline \multicolumn{7}{|c|}{ Manual activity capacity } \\
\hline & $\mathrm{D}$ & $36(35-36)$ & $34(4)$ & $100(97-100)$ & & $<.001$ \\
\hline & ND & $28(17-36)$ & $25(11)$ & $78(47-100)$ & & \\
\hline \multicolumn{7}{|l|}{ Body functions } \\
\hline \multirow[t]{9}{*}{ Mobility } & Thumb opposition & & & & & \\
\hline & $\mathrm{D}$ & $10(9-10)$ & $9(3)$ & NA & NA & $<.001$ \\
\hline & ND & $6(1-10)$ & $6(4)$ & & & \\
\hline & TAROM & & & & & \\
\hline & $\mathrm{D}$ & $760(661-1281)$ & $911(344)$ & NA & NA & .143 \\
\hline & ND & $693(292-1295)$ & $745(508)$ & & & \\
\hline & Palm abduction & & & & & \\
\hline & $\mathrm{D}$ & $48(34-54)$ & $42(19)$ & & 89 (39) & .058 \\
\hline & ND & $41(18-53)$ & $35(23)$ & & $71(47)$ & \\
\hline \multirow[t]{15}{*}{ Strength (Newton) } & Grip & & & & & \\
\hline & $\mathrm{D}$ & $160(120-210)$ & $165(84)$ & $89(71-107)$ & 87 (37) & $<.001$ \\
\hline & ND & $75(38-140)$ & $90(71)$ & $47(21-75)$ & $49(36)$ & \\
\hline & Tip-tip & & & & & \\
\hline & $\mathrm{D}$ & $30(20-40)$ & $31(16)$ & $77(59-91)$ & $73(33)$ & $<.001$ \\
\hline & ND & $20(40-60)$ & $20(16)$ & $54(0-77)$ & 47 (36) & \\
\hline & Tripod & & & & & \\
\hline & $\mathrm{D}$ & $40(30-50)$ & $42(20)$ & $79(60-90)$ & $75(32)$ & $<.001$ \\
\hline & ND & $20(0-40)$ & $22(22)$ & $43(0-65)$ & 40 (39) & \\
\hline & Key & & & & & \\
\hline & $\mathrm{D}$ & $50(40-60)$ & $51(25)$ & $80(67-99)$ & $79(33)$ & $<.001$ \\
\hline & ND & $30(0-40)$ & $31(26)$ & $56(0-76)$ & 48 (39) & \\
\hline & Opposition & & & & & \\
\hline & $\mathrm{D}$ & $54(46-63)$ & 54 (19) & $86(78-102)$ & $85(28)$ & .001 \\
\hline & ND & $47(0-57)$ & $36(27)$ & $74(0-91)$ & $59(43)$ & \\
\hline
\end{tabular}

and complete the task with a normal grip and motion. If relevant anatomical structures or body functions were absent, children could not score maximally on the task, but only a maximum of 2 out of 4 (see Table 2). All scores are added up and provide a sum score between 0 and 36. Based on this score the limitation of manual activity capacity can be qualified as severe (score $\leq 13$ ), moderate (score 14-21), and mild (score $\geq 22$ ).

\section{Body function}

The variables taken into account at the domain of body functions were the total active range-of-motion (TAROM), palmar abduction of the thumb, Kapandji thumb range-of-motion score, and grip-, tip-tip pinch-, tripod pinch-, lateral pinch- and opposition strength.

We evaluated joint mobility using a finger goniometer to calculate TAROM per hand as the sum of the AROM of all present joints. ${ }^{11}$ As a result, if joints or fingers were lacking, a lower TAROM was scored. Additionally, we evaluated thumb range of motion using Kapandji thumb range-of-motion, ${ }^{12}$ and palmar abduction using the Pollexograph. ${ }^{13}$ Muscle strength was evaluated by measuring grip- and pinch strength (tip-tip pinch, tripod pinch and lateral pinch) with the Lode handgrip and pinch grip dynamometer (Lode Medical Technology, Groningen, The Netherlands) and opposition strength was measured using the Rotterdam Intrinsic Hand Myometer (RIHM) ${ }^{14-16}$ The mean force of 3 maximum voluntary contractions was recorded for all strength measurements. In addition, we used the reference values for grip and pinch strength for children reported by Surrey et al and Molenaar et al to express the forces as a percentage of the reference values. ${ }^{15,17}$

To correct for severity of CHD, we added the number of affected digits per hand and affected sides (unilateral involvement versus bilateral involvement) as possible covariates. In addition, hand dominance was taken into account as a covariate in the model.
Hand dominance was established by taking the writing hand as the dominant hand. Both manual activity capacity examination and body functions measurements were evaluated for both hands.

\section{Statistical analysis}

Measures of centrality and spread on all outcome measures per hand are presented in Table 3. Differences between dominant hands and nondominant hands were tested with the Friedman's test. A mixed model of 212 hands was constructed to determine whether the Eliasson score was related to the covariates. Intrapatient correlation in the Eliasson score was accounted for by taking all dominant and nondominant hands (not only the affected hands) as random effect in the model. The initial model consisted of all the covariates as fixed effects together with the interaction terms between the covariates and the effect of hand dominance. From the full model non-significant fixed effects were removed stepwise using likelihood ratio test with $p$-values for removal $>$.1. The main fixed effects were kept in a final model if the interaction term was significant and are displayed in Table 4 . The need for the random effect was tested using a mixture of chi-square distributions.

\section{Results}

The distribution of the scores on manual activity capacity is displayed in Fig. 1. Only 8 unilaterally affected children were affected at the dominant hand with no or mild limitation of manual activity capacity. Limitation of manual activity capacity of the other unilaterally affected children's nondominant hand was almost equally spread over all severity categories (Fig. 1a). In the bilaterally affected children, the nondominant hand was more limited in manual activity capacity than the dominant hand (Fig. 1b).

Table 3 represents measures of central tendency and statistical significance of all outcome measures. Despite of the large variance 
Table 4

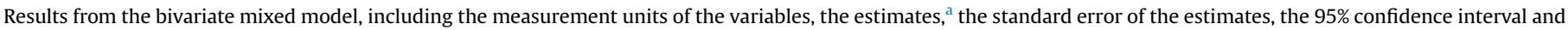
the significance

\begin{tabular}{|c|c|c|c|c|c|c|}
\hline Outcome variable & Fixed effects & Units & Estimate & Std. error & 95\% confidence interval & $p$-value \\
\hline \multirow[t]{13}{*}{ Manual activity capacity } & Intercept dominant hand & & 28.9 & 1.49 & $26.0,31.8$ & $<.001$ \\
\hline & Intercept nondominant hand & NA & 8.9 & 1.57 & $5.8,12.0$ & $<.001$ \\
\hline & Bilateral involvement vs unilateral involvement & NA & -3.8 & .67 & $-5.1,-2.5$ & $<.001$ \\
\hline & Number of affected fingers & & -.6 & .17 & $-.9,-.3$ & .001 \\
\hline & TAROM & Degrees & .002 & .0009 & $.0002, .0038$ & .029 \\
\hline & Palmar abduction & Degrees & .05 & .02 & $.01, .09$ & .001 \\
\hline & Grip strength & $\%$ of norm & .005 & .01 & $-.01, .03$ & .616 \\
\hline & Tip pinch & $\%$ of norm & .03 & .01 & $.01, .05$ & .026 \\
\hline & Lateral pinch & $\%$ of norm & .02 & .01 & $.0004, .04$ & .034 \\
\hline & Nondominant $*$ TAROM & NA & .008 & .001 & $.0006, .0010$ & $<.001$ \\
\hline & Nondominant $*$ palmar abduction & NA & .07 & .03 & $.01, .13$ & .005 \\
\hline & Nondominant $*$ bilateral involvement & NA & 4.8 & 1.1 & $2.6,7.0$ & $<.001$ \\
\hline & Nondominant $*$ grip strength & NA & .07 & .02 & $.03, .11$ & $<.001$ \\
\hline
\end{tabular}

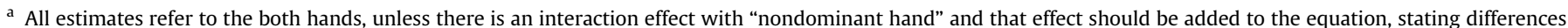
between dominant and nondominant hands.

within both dominant and nondominant hands on all outcome measures at body function level, dominant hands scored significantly better on all body functions than nondominant hands (Table 3). On average, children with CHD showed reduced muscle strength on all strength measurements compared to reference values and differences were more evident in the nondominant hand. Bilateral involvement lowered the score on manual activity capacity, as did the number of affected fingers.

\section{Relations between manual activity capacity and hand dominance}

The scatterplots of the explaining variables versus manual activity capacity are shown in Fig. 2a-e. The nondominant hand scored lower on manual activity capacity than the dominant hand, with the difference being larger on the left side of the scatterplots, i.e. when the scores on the body functions variables are lower. However, when adjusting for the effects of other covariates in the model (Table 4), the largest effect was found for hand dominance $(p<.001)$. More specifically, in children with low scores on body functions, the difference between the dominant and nondominant hand can add up to 20 points (i.e. the estimate of the intercept of the dominant hand minus the intercept of the nondominant hand, Table 4). The interaction effect between "nondominant" and "bilateral involvement" indicates that the effect of bilateral involvement on the manual activity capacity was different for dominant and nondominant hand. In comparison with unilaterally affected children, the dominant hand of bilaterally affected children scored 3.8 points lower on manual activity capacity and the nondominant hand of bilaterally affected children scores 1 point (4.8-3.8) better than the nondominant hand of unilaterally affected children (Table 4).

\section{Relations between manual activity capacity and range of motion}

Smaller effects were found for TAROM, palmar abduction, grip-, tip-tip- and lateral pinch strength and number of affected digits. While hand dominance and TAROM interact on estimating manual activity capacity, the effect of TAROM on manual activity capacity was different for the dominant and nondominant hand. Namely, for the nondominant hand the increase is larger by .008 per degree

a

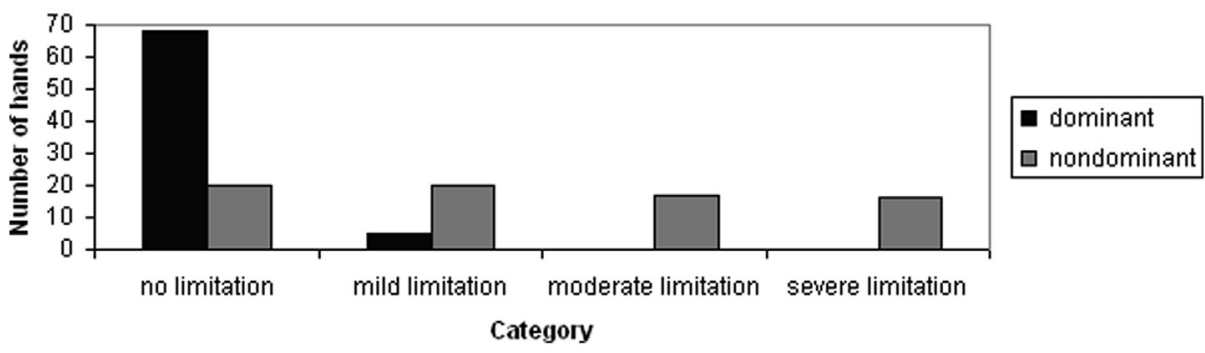

b

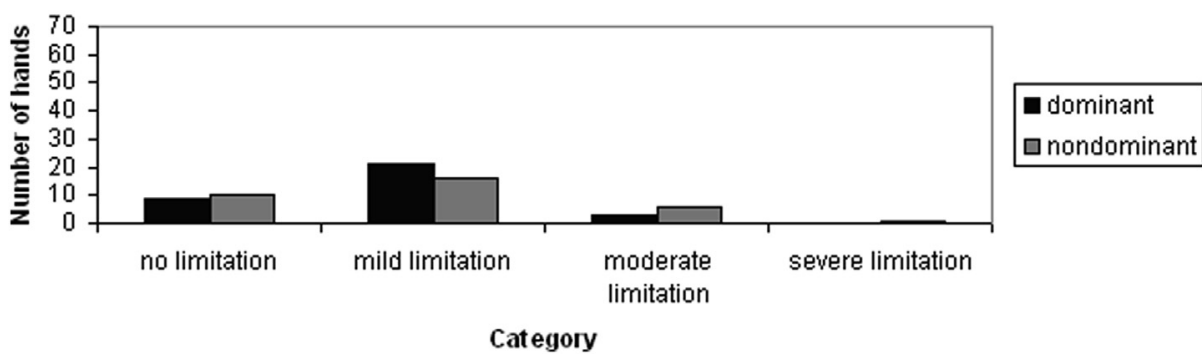

Fig. 1. a) Distribution of manual activity capacity of unilaterally affected children. b) Distribution of manual activity capacity of bilaterally affected children. 
a

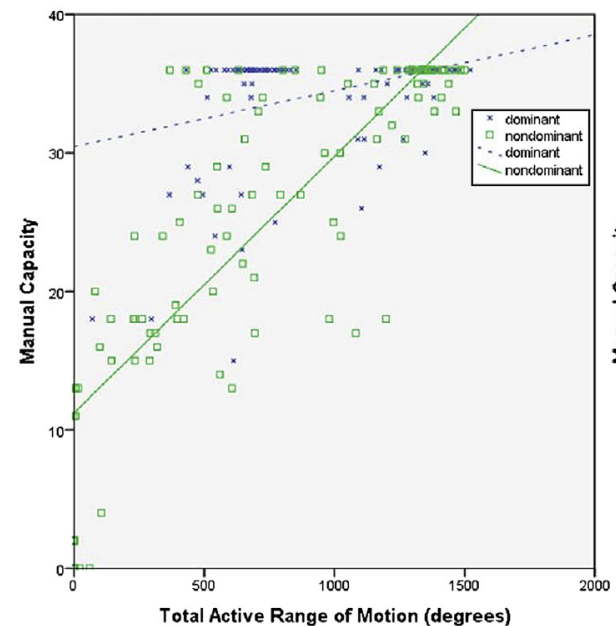

b

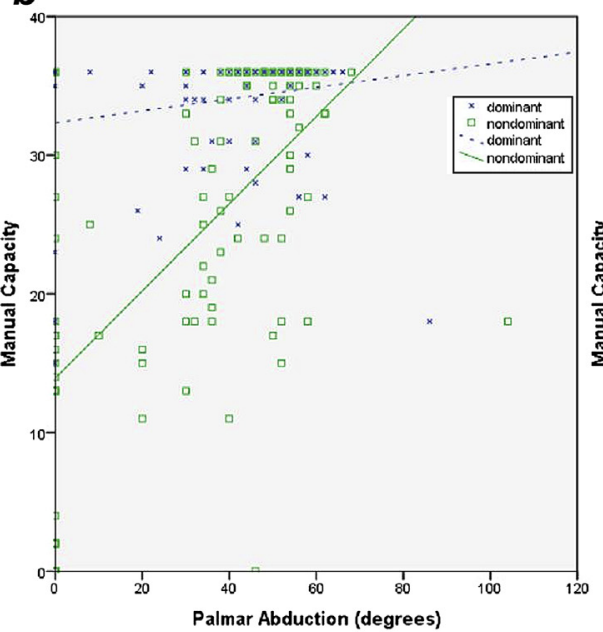

c

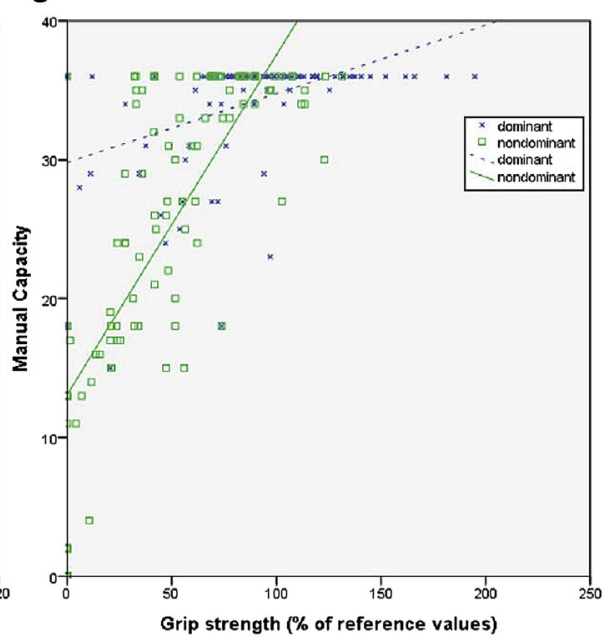

d

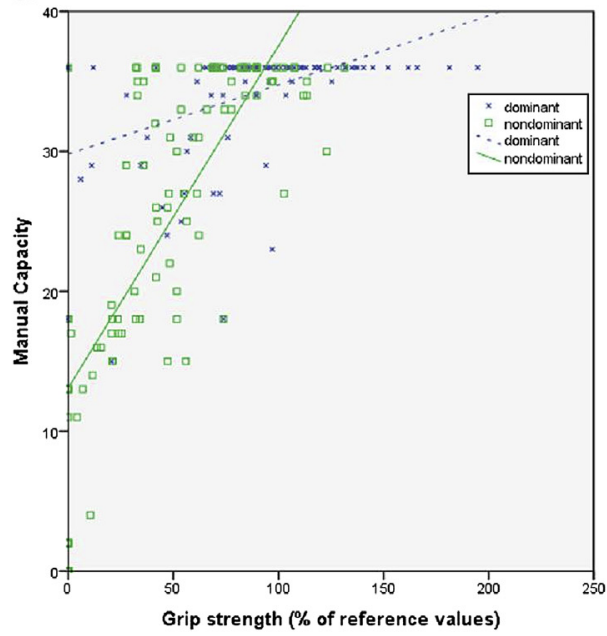

e

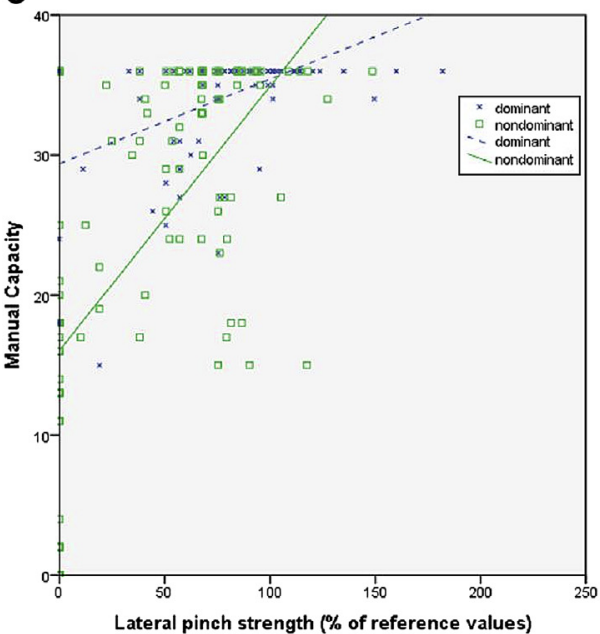

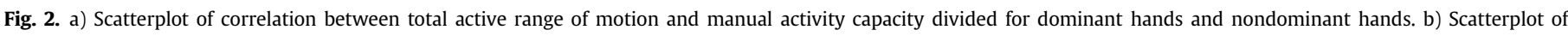

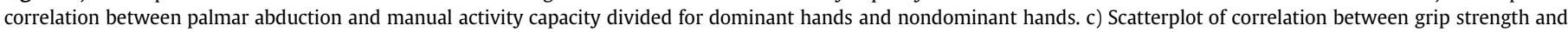

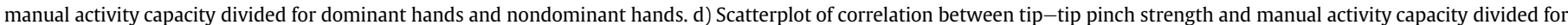

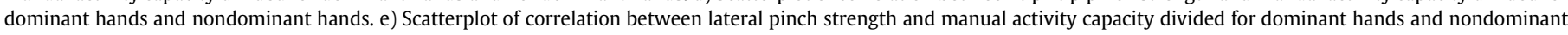
hands.

increase in TAROM. Since the scoring range on TAROM is about 1400 degrees, the difference of TAROM on the manual activity capacity score between the lowest and the highest TAROM score for the dominant hand is 2.8 points and for the nondominant hand 14 points on manual activity capacity. This interaction effect was also found with palmar abduction. The increase in manual activity capacity score per degree increase in palmar abduction for the nondominant hand is larger by .07. Since the scoring range on palmar abduction is $0^{\circ}-60^{\circ}$ the difference in manual activity capacity between the lowest and the highest score on palmar abduction is for the dominant hand 3 points and for the nondominant hand 7.2 points.

\section{Relations between manual activity capacity and muscle strength}

For grip strength, 1\% increase in strength enlarges the score with .075 points in the nondominant hand group on manual activity capacity (Table 4 ). With a range of $0-100 \%$ grip strength, this means a maximum difference of 7.5 points on the Eliasson test. For tip-tip pinch strength, the score increases with .03 points on manual activity capacity with a $1 \%$ increase in relative strength for both dominant and nondominant hands. This means a $100 \%$ strength is associated with a 3-point increase in manual activity capacity. The effect of lateral pinch is also the same for dominant and nondominant hands, namely .02 per 1 percent increase in relative strength. This means that the largest difference between the worst and the best scoring child on pinch strength is 2 points. An increasing number of affected digits have a negative effect on the manual activity capacity score. Each additionally affected digit decreases the score by .6 points for both the dominant and nondominant hand.

\section{Discussion}

In this study, we investigated limitation of manual activity capacity and its relation with body functions in 106 children with diverse forms of CHD. Manual activity capacity, measured with the Eliasson functional handgrip test, showed mild to moderate limitation. We found that manual activity capacity in these children is to great extent determined by hand dominance. Hand dominance also interacts with TAROM, palmar abduction of the thumb and grip strength in estimating manual activity capacity. Therefore, the effect of these body functions on manual activity capacity is different 
for the dominant hand than for the nondominant hand. While "bilateral involvement" interacts with hand dominance, the effect of hand dominance is different for unilaterally than bilaterally involved children. Due to the positive relation between grip-, tiptip pinch-, and lateral pinch strength and manual activity capacity, higher strength scores result in better manual activity capacity. More affected digits per hand have a negative effect on the manual activity capacity scores.

Measuring body functions in children with CHD is generally accepted for diagnostic purposes and for evaluating interventions. Although interest in manual activity capacity is growing, this is a less frequently-used outcome measure than measures regarding body functions. Manual activity capacity of children with a CHD of different severity, as measured in our study, is to our knowledge not previously reported. Since there is no well-defined score or classification to express disease severity in CHD and its subgroups, in the regression model, we have taken into account objective measures of severity, such as unilateral or bilateral involvement and number of affected digits, but also TAROM and muscle strength are indicators for severity. Correcting for these measures of severity, the regression analysis then allowed us the study the individual contributions of these body functions on manual activity capacity.

In this study, we included a relatively heterogenous population of children with CHD, with differences that varied from a simple syndactyly that will have little impact on body functions, to severe forms of radial deficiency that lead to very severe impairments in body functions. Consequently, based on the present findings, we cannot make statements for specific diagnosis groups. On the other hand, however, the large and diverse study population enabled us to study body functions, manual activity capacity and their interrelationships and determinants regardless of the diagnosis. These interactions otherwise could not be studied due to the small group sizes per diagnosis and even within these groups all subforms of CHD and its different comorbidities.

Hand dominance was the strongest predictor in our model, which means that if all other variables in the model are kept constant, hand dominance predicted most strongly the variance in manual activity capacity (i.e., when impairments are similar in both hands, dominant hands have a better manual activity capacity than nondominant hands). It should be noted, however, that hand dominance is largely debated ${ }^{18-20}$ and a difficult concept to describe, especially in case of CHD. Although hand dominance has been described as the tendency to perform the majority of tasks with one hand rather than the other, this does not necessarily mean that the chosen hand is more efficient. The distinction between hand dominance and hand performance has been extensively studied and conflicting theories are used to describe the development of the dominant hand. ${ }^{21,22}$ Some researchers describe that children choose their best performing hand to be their preferred or dominant hand, ${ }^{22}$ whereas others conclude that preference precedes performance. ${ }^{21}$ In the first model, the preferred hand in children with unilateral CHD will generally be the unaffected hand and in children with bilateral CHD the less-affected hand. In the second model, the child's preferred hand could be either hand, regardless of the impairment. In this study, since we did not expect hand dominance to be a major factor in determining the relation between manual activity capacity and body functions and for the time burden of the children, we choose to define the dominant hand by asking the children about their writing hand instead of a questionnaire. Now that we found that dominance played a major role in relation between the two levels, in retrospect we better might have chosen a questionnaire, enabling us to make firmer statements on hand dominance.

In our institution, surgeons often choose in bilaterally affected children to operate on the dominant hand first. The idea is that this makes the dominant hand stronger and better in manual activity capacity to perform daily activities. Sometimes children and their parents do not opt for a second operation due to experiences with the first operation. Therefore, it is crucial to choose the best option the first time. This depends also on the goal of the intervention. Based on our findings we would suggest that there is more to gain in manual activity capacity from body functions in the nondominant hand.

In this study, we found that when we compare children with similar scores on all body functions, the nondominant hand of bilaterally affected children scored better on manual activity capacity. This difference may be explained by the alternate use of both hands in bilaterally affected children in unimanual daily tasks while unilaterally involved children may only use the non-affected hand. As a result, the nondominant hand of bilaterally affected children may be more trained than that of unilaterally affected children. This phenomenon is referred to as developmental disregard in children with cerebral palsy and as learned non-use in other groups of unilaterally affected children and in adult stroke patients. ${ }^{23,24}$ If this is the case, forced use techniques or bimanual training could be an option for treatment in these children and needs to be further investigated since these therapy options have already proven to be effective in children with cerebral palsy and brachial plexus lesions. $^{25,26}$

Although their contribution was less explicit than hand dominance and severity of the CHD, several body functions also influenced manual activity capacity: TAROM, palmar abduction of the thumb, grip-, tip-tip pinch-, and lateral pinch strength. The effect of the body functions on manual activity capacity was larger for the nondominant hand than for the dominant hand. The univariate relations of all body functions with manual activity capacity seem to have a large effect in the nondominant hand (Fig. 2a-e), but when they are entered in the multivariate regression their effect is significant, but smaller.

Surgeons improve body functions aiming at an increase in manual activity capacity, but, based on our findings, this improvement in body functions may result in only small improvements in manual activity capacity. Children showed an average of $15-27 \%$ impairment in the dominant hand compared to typically developing children, but $0-3 \%$ limitation of manual activity capacity. For the non-dominant hand, an average difference of $29-60 \%$ in body functions was found compared to reference values but only a decrease in manual activity capacity of $22 \%$ as measured with the test of Eliasson et al Since the Eliasson test has a limited scale, it may have restricted finding stronger relations between manual activity capacity and body functions. On the other hand, this phenomenon is also found in other diagnosis groups. ${ }^{27,28}$ Even when all body functions that enhance manual activity capacity score 0 preoperatively and maximum postoperatively, the maximum increase in manual activity capacity for the dominant hand may be 11 points. On the other hand, for the nondominant hand this all may add up to 33 points on a scale from 0 to 36 .

Manual activity capacity may be higher in our study group compared to all children with CHD, because we also included children that underwent surgery in the past. This may influence the generalizability of the outcome on manual activity capacity for all children with CHD, but does not hinder the analysis of the relation between manual activity capacity and body functions.

In clinical practice, opposition strength is often mentioned as a key variable and several surgical techniques are practiced to enhance thumb opposition strength. However, in this study, we found that this variable did not significantly influence manual activity capacity in a multi-variate analysis. This means that it does not significantly contribute to manual activity capacity, when corrected for other variables. However, this does not mean that 
opposition is not important in functioning of the hand. For instance, for tip-tip pinch besides a stable thumb a child needs good opposition strength. This should be further investigated whether there is a difference in manual activity capacity pre- and postoperatively in children that underwent opposition strengthening.

\section{Conclusion}

In conclusion, the present study shows that in children with CHD the dominant hand scores better on manual activity capacity, even after correcting for differences in hand functions (e.g., strength and mobility) and that there is a stronger relationship between body function and manual activity capacity in non-dominant hands of bilaterally affected children.

\section{References}

1. Ho ES, Clarke HM. Functional evaluation in children with congenital upper extremity malformations. Clin Plast Surg. 2005 Oct;32(4):471-483. v.

2. WHO. International Classification of Functioning, Disability and Health, Child and Youth Version (ICF-CY). Geneva. 2007.

3. Arnould C, Penta M, Thonnard JL. Hand impairments and their relationship with manual ability in children with cerebral palsy. J Rehabil Med. 2007 Nov;39(9):708-714.

4. van Meeteren J, van Rijn RM, Selles RW, Roebroeck ME, Stam HJ. Grip strength parameters and functional activities in young adults with unilateral cerebra palsy compared with healthy subjects. J Rehabil Med. 2007 Oct;39(8):598-604.

5. Sakzewski L, Ziviani J, Boyd R. The relationship between unimanual capacity and bimanual performance in children with congenital hemiplegia. Dev Med Child Neurol. 2010 Sep;52(9):811-816.

6. Blair WF, Shurr DG, Buckwalter JA. Functional status in ulnar deficiency J Pediatr Orthop. 1983 Feb;3(1):37-40.

7. Buffart LM, Roebroeck ME, Janssen WG, et al. Hand function and activity performance of children with longitudinal radial deficiency. J Bone Joint Surg Am. 2008 Nov;90(11):2408-2415.

8. Ceulemans L, Degreef I, Debeer P, De Smet L. Outcome of index finger pollicisation for the congenital absent or severely hypoplastic thumb. Acta Orthop Belg. 2009 Apr;75(2):175-180.

9. Verdan C. The reconstruction of the thumb. Surg Clin North Am. 1968 Oct;48(5): 1033-1061.

10. Eliasson AC, Ekholm C, Carlstedt T. Hand function in children with cerebral palsy after upper-limb tendon transfer and muscle release. Dev Med Child Neurol. 1998 Sep;40(9):612-621.

11. Hamilton GF, Lachenbruch PA. Reliability of goniometers in assessing finger joint angle. Phys Ther. 1969 May;49(5):465-469.
12. Kapandji IA. Clinical evaluation of the thumb's opposition. J Hand Ther. 1992;2: 102-106.

13. de Kraker M, Selles RW, Schreuders TA, Hovius SE, Stam HJ. The Pollexograph: a new device for palmar abduction measurements of the thumb. $J$ Hand Ther. 2009 Jul-Sep;22(3):271-276. quiz 7.

14. Molenaar HM, Selles RW, Schreuders TA, Hovius SE, Stam HJ. Reliability of hand strength measurements using the Rotterdam Intrinsic Hand Myometer in children. J Hand Surg Am. 2008 Dec;33(10):1796-1801.

15. Molenaar HM, Selles RW, Willemsen SP, Hovius SE, Stam HJ. Growth diagrams for individual finger strength in children measured with the RIHM. Clin Orthop Relat Res. 2011 Mar:469(3):868-876.

16. Molenaar HM, Zuidam JM, Selles RW, Stam HJ, Hovius SE. Age-specific reliability of two grip-strength dynamometers when used by children. J Bone Joint Surg Am. 2008 May;90(5):1053-1059.

17. Surrey LR, Hodson J, Robinson E, et al. Pinch strength norms for 5-to 12-yearolds. Phys Occup Ther Pediatr. 2001;21(1):37-49.

18. Brown SG, Roy EA, Rohr LE, Bryden PJ. Using hand performance measures to predict handedness. Laterality. 2006 Jan;11(1):1-14.

19. Corbetta D, Williams J, Snapp-Childs W. Plasticity in the development of handedness: evidence from normal development and early asymmetric brain injury. Dev Psychobiol. 2006 Sep;48(6):460-471.

20. Corbetta D, Thelen E. Lateral biases and fluctuations in infants' spontaneous arm movements and reaching. Dev Psychobiol. 1999 May;34(4):237-255.

21. Mc Manus IC, Bryden MP. The Genetics of Handedness, Cerebral Dominance and Lateralization. In: Rapin I, Segalowitz S, eds. Handbook of Neuropsychology Developmental Neuropsychology. Amsterdam: Elsevier; 1992.

22. Annett M. Left, Right, Hand and Brain: The Right Shift Theory. Hillsdale, NJ: LEA 1985.

23. Houwink A, Aarts PB, Geurts AC, Steenbergen B. A neurocognitive perspective on developmental disregard in children with hemiplegic cerebral palsy. Res Dev Disabil. 2011 Nov-Dec;32(6):2157-2163.

24. Michielsen ME, Selles RW, Stam HJ, Ribbers GM, Bussmann JB. Quantifying Nonuse in Chronic stroke patients: a study into Paretic, Nonparetic, and bimanual upper-limb Use in daily life. Arch Phys Med Rehabil. 2012 Nov;93(11): 1975-1981.

25. Aarts PB, Jongerius PH, Geerdink YA, van Limbeek J, Geurts AC. Effectiveness of modified constraint-induced movement therapy in children with unilatera spastic cerebral palsy: a randomized controlled trial. Neurorehabil Neural Repair. 2010 Jul-Aug;24(6):509-518.

26. Hoare B, Imms C, Carey L, Wasiak J. Constraint-induced movement therapy in the treatment of the upper limb in children with hemiplegic cerebral palsy: a Cochrane systematic review. Clin Rehabil. 2007 Aug;21(8):675685

27. Chiu HC, Ada L, Butler J, Coulson S. Relative contribution of motor impairments to limitations in activity and restrictions in participation in adults with hemiplegic cerebral palsy. Clin Rehabil. 2010 May;24(5):454-462.

28. Vandervelde L, Van den Bergh PY, Renders A, Goemans N, Thonnard JL. Relationships between motor impairments and activity limitations in patients with neuromuscular disorders. J Neurol Neurosurg Psychiatry. 2009 Mar;80(3): $326-332$. 


\section{JHT Read for Credit \\ Quiz: \#317}

Record your answers on the Return Answer Form found on the tear-out coupon at the back of this issue or to complete online and use a credit card, go to JHTReadforCredit.com. There is only one best answer for each question.

\#1. For bilaterally involved patients the authors recommend
a. doing surgery on the dominant extremity first
b. doing surgery on the non-dominant extremity first
c. doing no surgery
d. doing surgery on both extremities at the same time

\#2. The authors use the definition of body functions as described by the
a. ASHT
b. HTCC
c. Dutch Medical Association
d. WHO's ICF-CY

\#3. The relationship between capacity and function is
a. not addressed in this article
b. intuitively obvious
c. not straight forward
d. linear

\#4. The authors found that opposition strength
a. was high on the list of priorities to improve
b. did not affect function as much as traditionally thought
c. should be addressed in the dominant hand first
d. did not need to be addressed

\#5. To fully grasp this article one must know the difference between capacity and performance
a. true
b. false

When submitting to the HTCC for re-certification, please batch your JHT RFC certificates in groups of 3 or more to get full credit. 